\title{
Effects of infections with five sexually transmitted pathogens on sperm quality
}

\author{
Sung Jae Kim', Doo-Jin Paik', Joong Shik Lee², Hyo Serk Lee², Ju Tae Seo², Mi Seon Jeong ${ }^{3}$, Jae-Ho Lee ${ }^{4}$, Dong Wook Park', \\ Sangchul Han ${ }^{5}$, Yoo Kyung Lee ${ }^{6}$, Ki Heon Lee ${ }^{6}$, In Ho Lee ${ }^{6}$, Kyeong A So ${ }^{6}$, Seon Ah Kim ${ }^{6}$, Juree Kim7, Tae Jin Kim ${ }^{6}$ \\ 'Department of Anatomy · Cell Biology, Hanyang University College of Medicine, Seoul; ${ }^{2}$ Department of Urology, ${ }^{3}$ Laboratory of Research and \\ Development for Genomics, ${ }^{4}$ Laboratory of Molecular Oncology, ${ }^{5}$ Laboratory of Reproductive Medicine, ${ }^{6}$ Department of Obstetrics and Gynecology, \\ and 'Department of Radiation Oncology, Cheil General Hospital and Women's Healthcare Center, Dankook University College of Medicine, Seoul, Korea
}

Objective: This study investigated the prevalence of infections with human papillomavirus, Chlamydia trachomatis, Ureaplasma urealyticum, Mycoplasma hominis, and Mycoplasma genitalium in the semen of Korean infertile couples and their associations with sperm quality.

Methods: Semen specimens were collected from 400 men who underwent a fertility evaluation. Infection with above five pathogens was assessed in each specimen. Sperm quality was compared in the pathogen-infected group and the non-infected group.

Results: The infection rates of human papillomavirus, C. trachomatis, U. urealyticum, M. hominis, and M. genitalium in the study subjects were $1.57 \%, 0.79 \%, 16.80 \%, 4.46 \%$, and $1.31 \%$, respectively. The rate of morphological normality in the $U$. urealyticum-infected group was significantly lower than in those not infected with $U$. urealyticum. In a subgroup analysis of normozoospermic samples, the semen volume and the total sperm count in the pathogen-infected group were significantly lower than in the non-infected group.

Conclusion: Our results suggest that infection with $U$. urealyticum alone and any of the five sexually transmitted infections are likely to affect sperm morphology and semen volume, respectively.

Keywords: Chlamydia trachomatis; Human papillomavirus; Mycoplasma genitalium; Mycoplasma hominis; Semen analysis; Sexually transmitted disease; Ureaplasma urealyticum

\section{Introduction}

Sexually transmitted infections (STIs) are a major global concern, and approximately 340 million new cases of infections occur annual-

Received: Aug 3, 2017· Revised: Nov 15, 2017· Accepted: Nov 29, 2017

Corresponding author:Tae Jin Kim

Department of Obstetrics and Gynecology, Cheil General Hospital and Women's Healthcare Center, Dankook University College of Medicine, 17 Seoae-ro 1-gil, Jung-gu, Seoul 04619, Korea

Tel: +82-2-2000-7577 Fax:+82-2-2000-7183 E-mail: kimonc111@naver.com

*This work was supported in part by a grant from the investigator-initiated studies program of Merck Sharp \& Dohme Corp., the Korean Healthcare Technology R\&D

Project, the Ministry of Health \& Welfare, the Republic of Korea

(A10206510111250100), and Basic Science Research Programs through the National Research Foundation of Korea funded by the Ministry of Education, Science and Technology (NRF-2012R1A1A4A01014504).

This is an Open Access article distributed under the terms of the Creative Commons Attribution Non-Commercial License (http://creativecommons.org/licenses/by-nc/4.0/) which permits unrestricted non-commercial use, distribution, and reproduction in any medium, provided the original work is properly cited. ly worldwide [1]. More than 30 different bacteria, viruses, and parasites have been identified as sexually transmitted pathogens (STPs) [2]. Human papillomavirus (HPV), Chlamydia trachomatis, Ureaplasma urealyticum, Mycoplasma hominis, and Mycoplasma genitalium are the most prevalent viral and bacterial STPs that cause no or only mild symptoms in the majority of cases. Only repeated or persisting infections of these five STPs are considered to cause clinical symptoms [2].

$\mathrm{HPV}$ is a non-enveloped double-stranded DNA virus that causes the most common STI. More than $100 \mathrm{HPV}$ types have been identified, including over 40 anogenital types [3]. HPV infection is a concern in men not only because it is the main risk factor for the carcinogenesis of penile cancer and genital warts [4], but also because semen is the main vector for horizontal (male to female) and vertical (father to fetus) transmission of the virus. According to some studies, HPV infection in sperm cells might be associated with reduced sperm motility 
and asthenozoospermia $[5,6]$.

Bacterial, mycological, and protozoal STls are known to be curable with appropriate antibiotic agents [2]. One of the most prevalent bacterial STPs is C. trachomatis. C. trachomatis is a Gram-negative bacterium and has a circular genome of 1,042 kbp. The World Health Organization (WHO) estimated that over 90 million new C. trachomatis infections occur per year [7]. Several previous studies have been conducted of $C$. trachomatis in the serum or semen in relation to reduced pregnancy rates and impaired sperm quality $[8,9]$. Chlamydial infection may contribute to pelvic inflammatory disease, which is a major health concern associated with reproductive health problems [10].

Hosseinzadeh et al. [11] suggested that coincubation of human spermatozoa with C. trachomatis caused premature sperm death. They also proposed that coincubation with C. trachomatis lipopolysaccharide induced caspase-mediated apoptosis in ejaculated human sperm [12]. In addition, Ljubin-Sternak and Mestrovic [7] described the role of $C$. trachomatis in male infertility, sperm quality, and in vitro fertilization outcomes, and suggested that infection with C. trachomatis is associated with impaired sperm quality.

The Mycoplasma family is the most frequent STP; they are the smallest living organisms, and this family includes a number of species [7]. The effects of infections with genital Mycoplasma species on sperm quality are still controversial and need to be elucidated. Xu et al. [13] suggested that a high percentage of $U$. urealyticum in the sperm of sexual partners increases secondary infertility and reduces sperm quality. It has also been suggested that the considerably higher incidence of $M$. hominis in patients with infertility causes abnormal semen patterns [14]. In addition, Al-Sweih et al. [15] reported that $M$. genitalium appeared to impair sperm quality.

Although it has been estimated that $15 \%$ of cases of male infertility are caused by STIs [2], the effect of infections with HPV, C. trachomatis, U. urealyticum, M. hominis, and M. genitalium on infertility is still unclear. Therefore, this study aimed to investigate the prevalence of HPV, C. trachomatis, U. urealyticum, M. hominis, and $M$. genitalium in the semen of Korean men who underwent an infertility workup and their effects on seminal variables, including semen volume, total sperm count, sperm concentration, motility, and morphological normality.

\section{Methods}

\section{Study subjects}

This study was approved by the ethical committee of Cheil General Hospital (No. CGH-IRB-2013-31) and written informed consent was obtained from all participants. To estimate the sample size, it was assumed that at least twice the number of HPV-infected sperm cells would have impaired sperm motility compared to HPV non-infected sperm cells, on the basis of a study by Foresta et al. [6], which revealed that $70 \%$ of HPV-infected sperm cells had impaired sperm motility, in comparison to $30 \%$ among non-infected sperm cells. The study would require 380 samples to achieve $80 \%$ power with a onesided alpha level of 0.05 . A $4 \%$ dropout rate was estimated, and 400 samples were planned. We enrolled 400 men who underwent a fertility evaluation at the Fertility Center of Cheil General Hospital and Women's Healthcare Center. The inclusion criteria were men who (1) were between 19 and 40 years old and (2) had sexual intercourse with a woman within the past year. The exclusion criteria were men who (1) were diagnosed with a sex gene abnormality; (2) were diagnosed with a serious structural disorder of the pelvic organs; (3) were diagnosed with the inability to ejaculate due to physical or mental disease; (4) had no experience in ejaculation by masturbation; (5) had a history of a male sterilization operation; (6) had a history of an STI within the previous year, genital warts, penile or anal cancer, testicular trauma, cryptorchidism, post-mumps orchitis, varicocele, or seminal infection; or (7) had penile discharge or unusual penile symptoms such as severe pain within the previous 6 months.

Subjects were withdrawn from the study for the following reasons: occurrence of a significant disease in the subject, the request of the subject himself, a protocol violation by the subject or the investigator, or the investigators' opinion that participation was required to be discontinued.

Infection with HPV, C. trachomatis, U. urealyticum, M. hominis, and M. genitalium was assessed in each specimen. Samples infected with any pathogen were assigned to the pathogen-infected group, those with no infection with any pathogen were assigned to the non-infected group.

\section{Sample collection}

Semen samples were collected by masturbation after a 3-day period of sexual abstinence. After liquefaction at room temperature, the semen samples were centrifuged ( $430 \times g, 10$ minutes) and the pellets were washed three times with sterile phosphate-buffered saline for fractionation of the sperm and seminal plasma. All samples were stored at $-20^{\circ} \mathrm{C}$ until they were processed and used for subsequent analyses. DNA was extracted from the purified sperm pellets with the QIAamp DNA kit or another DNA extraction kit (Qiagen, Gaithersburg, MD, USA) according to the manufacturer's instructions.

\section{HPV DNA chip test}

Using the guidelines of the Cheil HPV DNA chip kit (Cheil General Hospital and Women's Healthcare (enter, Seoul, Korea), an HPV DNA chip test was performed as described below. Isolated DNA was used for polymerase chain reaction (PCR). The primers for PCR were de- 
signed to detect 36 specific types of HPV $(6,11,16,18,30,31,32,33$, $35,39,40,42,43,44,45,51,52,53,54,55,56,58,59,62,66,67,68$ a, $68 \mathrm{~b}, 69,70,72,81,82,84,90$, and 91) (Supplement 1). Real-time PCR was conducted using a LightCycler 480 apparatus (Roche, Basel, Switzerland). The generated PCR product was denatured at $95^{\circ} \mathrm{C}$ for 10 minutes. The denatured PCR product with a hybridization buffer was added to the slide and incubated for hybridization at $60^{\circ} \mathrm{C}$ for 20 minutes. After hybridization, the slides were sequentially washed with two washing buffers (I and II: three times for 2 minutes each). The slides were dried and visualized by fluorescent scanning in a commercial DNA chip scanner, NimbleGen MS 200 (Tecan Austria, Grodig, Austria), and the HPV types were analyzed with GenePix Pro 6.0 software (Axon instruments Inc., Union City, CA, USA).

\section{Detection method for C. trachomatis, U. urealyticum, $M$. hominis, and M. genitalium}

A MYCOFAST Evolution 2 kit (International Microbio, Signes, France) was utilized to detect the presence of $C$. trachomatis, $U$. urealyticum, $M$. hominis, and $M$. genitalium. The test was a liquid microculture method using the patterns of resistance to six antibiotics (lincomycin, trimethoprim sulfamethoxazole, erythromycin, doxycycline, roxithromycin, and ofloxacin). Infection with a pathogen was indicated by a color change of the medium from yellow-orange to red.

\section{Semen analysis}

After liquefaction at room temperature, the semen volume, sperm concentration, total sperm count, motility, and morphological normality were determined by the WHO guidelines for semen analysis (fifth edition, 2010). Computer-aided sperm analysis was used to determine semen volume, sperm concentration, total sperm count, and motility. The lower limit of morphological normality was 4\%. Investigators involved in the sperm analysis and HPV subtype analysis were blinded to patient information.

\section{Statistical analysis}

The prevalence of infections with HPV, C. trachomatis, U. urealyticum, M. hominis, and M. genitalium, as well as the distribution of HPV genotypes, was analyzed. The potential effects of infection with the various pathogens on sperm quality (semen volume, total sperm count, sperm concentration, sperm motility, and morphological normality) were evaluated by the $t$-test for parametric comparisons or the Mann-Whitney $U$-test for nonparametric comparisons. The potential effects of infection with the various pathogens on sperm quality were evaluated by the chi-square test or the Fisher exact test. The Mann-Whitney test was used to compare the sperm quality of the pathogen-infected group with that of the non-infected group in normozoospermic samples, as well as to compare the seminal vari- ables of the pathogen-infected group with those of the non-infected group for $U$. urealyticum alone and for any pathogen, because the ratios of subjects between the pathogen-infected and non-infected groups were $>1-1.5$. All statistical analyses were performed using IBM SPSS ver. 21.0 (IBM Corp., Armonk, NY, USA). The $p$-values $<0.05$ were considered to indicate statistical significance.

\section{Results}

\section{Demographic characteristics}

Two of the 400 subjects who visited the Fertility Center of Cheil General Hospital and Women's Healthcare Center were excluded because their infection data were missing. Among the remaining 398 subjects, 86 were positive for at least one of the five pathogens (the pathogen-infected group) and 312 were negative for all five pathogens (the non-infected group). The participants' mean age was $34.98 \pm 4.44$ years for the pathogen-infected group $(n=86)$ and $35.44 \pm 4.52$ years for the non-infected group $(n=312)$. Azoospermia and necrozoospermia were present in 15 and two subjects, respectively. Nineteen subjects with missing data, azoospermia, or necrozoospermia were excluded from further analysis. Two of these 19 subjects were pathogen-infected, and they both had azoospermia.

A comparison of subjects' demographic information showed no significant difference in age (34.98 \pm 4.44 years vs. $35.44 \pm 4.52$ years) or the rates of azoospermia ( $2.32 \%$ vs. $4.17 \%)$ and necrozoospermia ( $0 \%$ vs. $0.64 \%$ ) between the pathogen-infected group and the noninfected group.

\section{Prevalence of the five STPs in the semen of Korean infertile couples}

Among the 381 subjects in the study, 74 were infected with a single pathogen and 10 were infected by multiple pathogens. Nine of the subjects in the multiple pathogen-infected group were infected by two pathogens (six U. urealyticum $+M$. hominis; two HPV+U. urealyticum; one M. hominis+M. genitalium) and one was infected by three pathogens (C. trachomatis $+U$. urealyticum $+M$. hominis).

Among the 381 semen samples, six were found to be positive for HPV infection, while 375 were found to be negative. Regarding the HPV type detected in semen, types 18,31,42,56, 84, and 91 were found in each subject, respectively. Three of these were high-risk types of HPV $(18,31$, and 56), while three were low-risk types (42, 84, and 91$)$.

In addition, of the 381 subjects in the study, 3, 64, 17, and 5 subjects were positive for $C$. trachomatis, $U$. urealyticum, $M$. hominis, and $M$. genitalium, respectively.

\section{Comparison of the rate of normal sperm quality}

The proportion of samples that had normal criteria for each of the 
Table 1. Comparison of the rates of normal parameters in the pathogen-infected and non-infected groups

\begin{tabular}{|c|c|c|c|c|c|c|}
\hline Variable & $\begin{array}{c}\text { HPV } \\
\text { non-infected } \\
(n=375) / \\
\text { infected }(n=6)\end{array}$ & $\begin{array}{c}\text { Uu } \\
\text { non-infected } \\
(n=317) / \\
\text { infected }(n=64)\end{array}$ & $\begin{array}{c}C t \\
\text { non-infected } \\
(n=378) / \\
\text { infected }(n=3)\end{array}$ & $\begin{array}{c}\text { Mh } \\
\text { non-infected } \\
(n=364) / \\
\text { infected }(n=17)\end{array}$ & $\begin{array}{c}M g \\
\text { non-infected } \\
(n=376) / \\
\text { infected }(n=5)\end{array}$ & $\begin{array}{l}\text { Any pathogen } \\
\text { non-infected } \\
(n=297) / \\
\text { infected }(n=84)^{a}\end{array}$ \\
\hline Semen volume & $92.0 / 100$ & $91.8 / 93.8$ & $92.1 / 100$ & $92.0 / 94.1$ & $96.5 / 100$ & $92.2 / 91.6^{b)}$ \\
\hline Total sperm count & $96.5 / 100$ & $96.5 / 96.9$ & $96.6 / 100$ & $96.4 / 100$ & $96.8 / 80.0$ & $96.6 / 96.4$ \\
\hline Sperm concentration & $97.3 / 100$ & $97.2 / 98.4$ & $97.6 / 100$ & $97.5 / 94.1$ & $97.3 / 100$ & 97.3/97.6 \\
\hline Sperm motility & $75.2 / 80.0$ & $75.1 / 76.6^{b)}$ & $75.1 / 100$ & $75.0 / 82.4$ & $75.0 / 100$ & $74.0 / 79.7^{\mathrm{b})}$ \\
\hline Sperm morphology & $42.7 / 33.3$ & $42.7 / 29.7^{\mathrm{b}, \mathrm{c}}$ & $42.6 / 33.3$ & $42.0 / 52.9^{b)}$ & $42.0 / 80.0$ & $44.7 / 33.3^{b)}$ \\
\hline Normozoospermia & $32.3 / 33.3$ & $34.4 / 21.9^{b)}$ & $32.3 / 33.3$ & $31.9 / 41.2^{b)}$ & $32.2 / 40.0$ & $34.0 / 23.8^{b)}$ \\
\hline
\end{tabular}

Values are presented as percent. The Fisher exact test was used for the comparison unless otherwise specified.

HPV, human papillomavirus; Uu, Ureaplasma urealyticum; Ct, Chlamydia trachomatis; Mh, Mycoplasma hominis; Mg, Mycoplasma genitalium.

${ }^{a}$ Any infection with $\mathrm{HPV}, \mathrm{Uu}, \mathrm{Ct}, \mathrm{Mh}$, or $\mathrm{Mg} ;{ }^{\text {b) }}$ Chi-square test; All $p$-values are not significant, except for ${ }^{\mathrm{c}} p<0.05$.

Table 2. Comparison of sperm quality in normozoospermic samples from the pathogen-infected group and the non-infected group

\begin{tabular}{lccc}
\hline Variable & Non-infected $(\mathrm{n}=101)$ & ${\text { Pathogen-infected }(\mathrm{n}=20)^{\mathrm{a})}}$ & $p$-value $\mathrm{e}^{\mathrm{b})}$ \\
\hline Semen volume $(\mathrm{mL})$ & $3.06 \pm 1.24$ & $2.52 \pm 0.78$ & $<0.05$ \\
Total sperm count $\left(\times 10^{6}\right)$ & $301.62 \pm 190.80$ & $213.29 \pm 98.02$ & $<0.05$ \\
Sperm concentration $\left(\times 10^{6} / \mathrm{mL}\right)$ & $97.12 \pm 50.79$ & $88.02 \pm 37.22$ & NS \\
Sperm motility $(\%)$ & $62.68 \pm 12.20$ & $62.36 \pm 10.66$ & NS \\
Sperm morphology $(\%)$ & $6.99 \pm 3.48$ & $7.65 \pm 3.60$ & NS \\
\hline
\end{tabular}

Values are presented as mean \pm standard deviation.

NS, not significant.

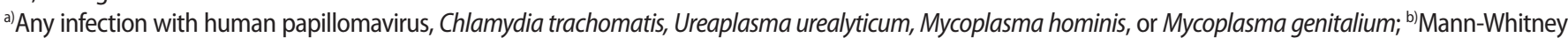
U-test.

five WHO parameters in each pathogen-infected group was compared to that in the corresponding non-infected group to clarify the effect of each pathogen on the proportion of samples that had normal criteria. The rate of normozoospermia in each pathogen-infected group was also compared to that of the corresponding non-infected group to clarify the effect of each pathogen on normozoospermia.

The $U$. urealyticum-infected group had a significantly lower rate of morphological normality than the $U$. urealyticum non-infected group $(p<0.05)$. However, the parameters of sperm quality in the HPV-, $C$. trachomatis-, M. hominis-, and M. genitalium-infected groups were not significantly different from those in the corresponding non-infected groups (Table 1).

\section{Comparison of semen parameters in subjects with normozoospermia}

A subgroup analysis of subjects with normozoospermia was conducted in order to investigate the impact of infection with these pathogens on sperm quality, given the possibility that cases with normozoospermia are less influenced by other confounding factors.

The five seminal variables of semen volume, total sperm count, sperm concentration, sperm motility, and morphology in normozoospermic subjects infected with any pathogen were compared to those in the non-infected group. The semen volume $(2.52 \pm 0.78 \mathrm{~mL}$ vs. $3.06 \pm 1.24 \mathrm{~mL}, p<0.05)$ and sperm count $\left(213.29 \pm 98.02 \times 10^{6}\right.$ vs. $\left.301.62 \pm 190.80 \times 10^{6}, p<0.05\right)$ were significantly lower in the pathogen-infected group than in the non-infected group (Table 2).

\section{Discussion}

Urogenital infections with HPV, C. trachomatis, U. urealyticum, $M$. hominis, and M. genitalium are among the most prevalent STls. The clinically silent natures of these five STPs have been explained as one of the main reasons for their high prevalence [2]. Even though infections with these five STPs have been associated with diverse clinical symptoms, their impact on sperm quality remains controversial.

Our findings indicate that the rates of infection with HPV, C. trachomatis, U. urealyticum, M. hominis, and M. genitalium were $1.57 \%$, $0.79 \%, 16.80 \%, 4.46 \%$, and $1.31 \%$, respectively. The prevalence of $U$. urealyticum was higher than that of the other pathogens, as has been observed in other studies $[15,16]$.

In previous studies, HPV prevalence in men ranged from $1.3 \%$ to $72.9 \%$, as multiple anatomic sites or specimens were assessed; 15 of those 25 studies (60\%) reported a HPV prevalence of more than $20 \%$ [17]. In particular, HPV was detected in $2.2 \%-41.3 \%$ of semen samples $[18,19]$. HPV infections were most commonly detected in the prepuce, and HPV-16 was the most commonly detected type [20]. 
Although the prevalence of pathogens varies according to the characteristics of the investigated population, including age and ethnicity, as well as different detection methods, the prevalence in this study was lower than that of other studies [21-24].

Several possible explanations can be proposed for the low prevalence, as follows. First, we excluded patients with warts around the sex organ or a history of STIs to minimize confounding factors. This exclusion criterion could be related to the low prevalence of pathogens. Second, the sensitivity of the detection systems used in this study may have been different from those used in other studies. We expect the impact of this possibility to have been minimal because both the Cheil HPV DNA chip and the MYCOFAST Evolution 2 Kit have been proven to be effective diagnostic assays. For example, the kappa value of the Cheil HPV chip was 0.963 (95\% confidence interval, 0.919-0.98), which is significantly higher than the value of 0.527 (95\% confidence interval, 0.447-0.59) of the conventional HPV DNA chip [25]. In addition, Park et al. [26] reported that the MYCOFAST Evolution 2 Kit had a sensitivity and specificity of $95 \%$ and $98 \%$, respectively.

It is also possible that the prevalence of these five STPs in the Korean population might be lower than in other communities. Shin et al. [27] reported that HPV was detected in $8.7 \%$ of male students in Korea, in contrast to 15 of 25 epidemiologic studies that reported HPV prevalence rates of more than $20 \%$ in other populations [28]. However, we cannot rule out other possibilities, including inconsistent sampling techniques, heterogeneous specimen types, and different processing methods, including the separation of semen into seminal plasma and sperm.

The low prevalence of pathogens in our findings may have been associated with the negative results of the comparisons of sperm quality according to infection with HPV, C. trachomatis, M. hominis, and M. genitalium (Table 1 and Supplement 2).

The relationship between infection with these five STPs and sperm quality remains unclear. Although some studies have found sperm quality to be impaired by infection with HPV [6], C. trachomatis [15], U. urealyticum [29], M. hominis [29], and M. genitalium [15], whether sperm quality was reduced by infection with HPV [30-32], C. trachomatis [33-35], U. urealyticum [35], and M. hominis [35,36] could not be clarified in other studies. We speculate that the following reasons may explain why infection with these pathogens did not influence sperm characteristics in some of our results (Supplement 2) and in some studies by other groups.

First, the conditions of the sperm quality tests could have been different among the tests in terms of the standardization of time analysis and in vivo human conditions. Standardizing the analysis and conditions with a sufficient number of infected sperm cells is therefore necessary to clarify the discrepancies among results. Second, it is possible that infection with these pathogens may have a minimal impact on sperm quality. This minimal impact might be lower than the threshold that can be detected, meaning that some investigators would not have been able to detect the impact of infection with these pathogens on sperm quality. Only persistent infection with a high pathogen load is likely to exceed the threshold that induces significant changes in sperm quality, whereas a low infection load is unlikely to exceed the threshold or to change the environment of the host sperm cells as suggested by the clinically silent nature of these conditions [2]. Third, as mentioned above, in the studies where infection with pathogens did not significantly influence sperm characteristics, it is possible that the infected sample size was too low, making it impossible to identify statistically significant findings. Finally, other unexplained factors might act as confounders, making it impossible to identify significant differences between the pathogen-infected and non-infected groups. In fact, one of the main limitations of this study is that we did not collect information from all participants regarding certain basal characteristics, such as body mass index, smoking, drinking, abstinence time, and frequency of sexual intercourse, all of which have been demonstrated to affect sperm quality and therefore could have acted as confounding factors. The possible relevance of other unexplained factors is supported by the following two facts: first, the prevalence of azoospermia in our study was $4.17 \%$ in the non-infected group and $2.32 \%$ in the infected group, while that of necrozoospermia in our study was $0.64 \%$ in the non-infected group and $0 \%$ in the infected group; and second, cases with less than $10 \%$ of the normal criterion for any indicator of sperm quality in our study accounted for $23.40 \%$ of the non-infected group and $17.44 \%$ of the infected group. Therefore, it is tempting to speculate that the Korean men who visited the infertility clinic might have had an innate reduced sperm quality, as reflected by their diagnosis with unexplained male infertility. Moreover, other possible confounding factors, including endocrine disorders, leukocytospermia, and unexplained factors could be involved in the reduction of sperm quality in those infected with pathogens such as HPV, U. urealyticum, C. trachomatis, M. hominis, and M. genitalium. For example, the endocrine disruption hypothesis proposed that endocrine disruptions during fetal, neonatal, and adult life might reduce sperm quality and reproductive hormone production [37]. Exclusion of these confounding factors would help clarify the relationship between these five STPs and sperm quality.

Therefore, a subgroup analysis of participants with normozoospermia was conducted, given the possibility that normozoospermic samples would be less influenced by other confounding factors. In this subgroup, the pathogen-infected group showed significantly lower semen volume $(p<0.05)$ and total sperm count $(p<0.05)$ than the non-infected group (Table 2). These results may suggest that in- 
fection with these five STPs is associated with impaired sperm quality. Male reproductive capacity has been found to be deficient in no fewer than $50 \%$ of infertile couples [38]. Additionally, approximately $40 \%$ of males from infertile couples were reported to have abnormal sperm quality [39]. These findings need to be confirmed in a largescale study with standard analyses and conditions.

Our results suggest that infection with $U$. urealyticum significantly decreased the rate of morphological normality of sperm. In addition, a trend was found for impaired semen volume in normozoospermic samples with $\mathrm{HPV}, C$. trachomatis, U. urealyticum, $M$. hominis and $M$. genitalium. Therefore, these data regarding the negative impact of these five STPs on sperm quality may support the necessity of testing Korean men for these five STPs.

\section{Conflict of interest}

This work was supported in part by a grant from the investigatorinitiated studies program of Merck Sharp \& Dohme Corp. The funders had no role in the design, collection, analysis or interpretation of this study. No other potential conflict of interest relevant to this article was reported.

\section{Supplementary materials}

Supplement 1. The primers for polymerase chain reaction. Supplemental data can be found at: http://ecerm.org/cerm-44-207-s001. pdf.

Supplement 2. Comparison of seminal variables in the pathogeninfected and non-infected groups. Supplemental data can be found at: http://ecerm.org/cerm-44-207-s002.pdf.

\section{References}

1. Centers for Disease Control and Prevention (CDC). STD-prevention counseling practices and human papillomavirus opinions among clinicians with adolescent patients: United States, 2004. MMWR Morb Mortal Wkly Rep 2006;55:1118-20.

2. World Health Organization. Sexually transmitted infections (STIs): fact sheet. Geneva: World Health Organization; 2016.

3. Schiffman M, Castle PE. Human papillomavirus: epidemiology and public health. Arch Pathol Lab Med 2003;127:930-4.

4. Gross G, Pfister H. Role of human papillomavirus in penile cancer, penile intraepithelial squamous cell neoplasias and in genital warts. Med Microbiol Immunol 2004;193:35-44.

5. Yang Y, Jia CW, Ma YM, Zhou LY, Wang SY. Correlation between HPV sperm infection and male infertility. Asian J Androl 2013;15: 529-32.
6. Foresta C, Noventa M, De Toni L, Gizzo S, Garolla A. HPV-DNA sperm infection and infertility: from a systematic literature review to a possible clinical management proposal. Andrology 2015;3:163-73.

7. Ljubin-Sternak S, Mestrovic T. Chlamydia trachomatis and genital mycoplasmas: pathogens with an impact on human reproductive health. J Pathog 2014;2014:183167.

8. Witkin SS, Kligman I, Bongiovanni AM. Relationship between an asymptomatic male genital tract exposure to Chlamydia trachomatis and an autoimmune response to spermatozoa. Hum Reprod 1995;10:2952-5.

9. Karinen L, Pouta A, Hartikainen AL, Bloigu A, Paldanius M, Leinonen $\mathrm{M}$, et al. Association between Chlamydia trachomatis antibodies and subfertility in the Northern Finland Birth Cohort 1966 (NFBC 1966), at the age of 31 years. Epidemiol Infect 2004; 132:977-84

10. Park ST, Lee SW, Kim MJ, Kang YM, Moon HM, Rhim CC. Clinical characteristics of genital Chlamydia infection in pelvic inflammatory disease. BMCWomens Health 2017;17:5.

11. Hosseinzadeh S, Brewis IA, Eley A, Pacey AA. Co-incubation of human spermatozoa with Chlamydia trachomatis serovar $\mathrm{E}$ causes premature sperm death. Hum Reprod 2001;16:293-9.

12. Eley A, Hosseinzadeh S, Hakimi H, Geary I, Pacey AA. Apoptosis of ejaculated human sperm is induced by co-incubation with Chlamydia trachomatis lipopolysaccharide. Hum Reprod 2005; 20:2601-7.

13. Xu C, Sun GF, Zhu YF, Wang YF. The correlation of Ureaplasma urealyticum infection with infertility. Andrologia 1997;29:219-26.

14. Lee JS, Kim KT, Lee HS, Yang KM, Seo JT, Choe JH. Concordance of Ureaplasma urealyticum and Mycoplasma hominis in infertile couples: impact on semen parameters. Urology 2013;81:121924.

15. Al-Sweih NA, Al-Fadli AH, Omu AE, Rotimi VO. Prevalence of Chlamydia trachomatis, Mycoplasma hominis, Mycoplasma genitalium, and Ureaplasma urealyticum infections and seminal quality in infertile and fertile men in Kuwait. J Androl 2012;33: 1323-9.

16. Rodriguez R, Hernandez R, Fuster F, Torres A, Prieto P, Alberto J. Genital infection and infertility. Enferm Infecc Microbiol Clin 2001;19:261-6.

17. Dunne EF, Nielson CM, Stone KM, Markowitz LE, Giuliano AR. Prevalence of HPV infection among men: a systematic review of the literature. J Infect Dis 2006;194:1044-57.

18. Foresta C, Garolla A, Zuccarello D, Pizzol D, Moretti A, Barzon L, et al. Human papillomavirus found in sperm head of young adult males affects the progressive motility. Fertil Steril 2010;93:802-6.

19. Palefsky JM. Human papillomavirus-related disease in men: not 
just a women's issue. J Adolesc Health 2010;46(4 Suppl):S12-9.

20. Nielson CM, Flores R, Harris RB, Abrahamsen M, Papenfuss MR, Dunne EF, et al. Human papillomavirus prevalence and type distribution in male anogenital sites and semen. Cancer Epidemiol Biomarkers Prev 2007;16:1107-14.

21. Gimenes F, Souza RP, Bento JC, Teixeira JJ, Maria-Engler SS, Bonini MG, et al. Male infertility: a public health issue caused by sexually transmitted pathogens. Nat Rev Urol 2014;11:672-87.

22. Brookings C, Goldmeier D, Sadeghi-Nejad H. Sexually transmitted infections and sexual function in relation to male fertility. Korean J Urol 2013;54:149-56.

23. Huang C, Zhu HL, Xu KR, Wang SY, Fan LQ, Zhu WB. Mycoplasma and Ureaplasma infection and male infertility: a systematic review and meta-analysis. Andrology 2015;3:809-16.

24. Liu J, Wang Q, Ji X, Guo S, Dai Y, Zhang Z, et al. Prevalence of Ureaplasma urealyticum, Mycoplasma hominis, Chlamydia trachomatis infections, and semen quality in infertile and fertile men in China. Urology 2014;83:795-9.

25. Seo HH, Kim YJ, Jeong MS, Hong SR, Lee IH, So KA, et al. Combined SYBR green real-time polymerase chain reaction and microarray method for the simultaneous determination of human papillomavirus loads and genotypes. Obstet Gynecol Sci 2016; 59:489-97.

26. Park HR, Kim YH, Lee HJ, Oh JS, Kim HJ. Usefulness of the MYCOFAST test (MYCOFAST ${ }^{\circledR}$ Evolution 2) for the diagnosis of nongonococcal genitourinary infections. Korean J Urol 2006;47: 1117-23.

27. Shin HR, Franceschi S, Vaccarella S, Roh JW, Ju YH, Oh JK, et al. Prevalence and determinants of genital infection with papillomavirus, in female and male university students in Busan, South Korea. J Infect Dis 2004;190:468-76.

28. Castellsague X. Natural history and epidemiology of HPV infection and cervical cancer. Gynecol Oncol 2008;110(3 Suppl 2):S4-7.

29. Huang C, Long X, Jing S, Fan L, Xu K, Wang S, et al. Ureaplasma urealyticum and Mycoplasma hominis infections and semen quality in 19,098 infertile men in China. World J Urol 2016;34: 1039-44.

30. Brossfield JE, Chan PJ, Patton WC, King A. Tenacity of exogenous human papillomavirus DNA in sperm washing. J Assist Reprod Genet 1999;16:325-8.

31. Connelly DA, Chan PJ, Patton WC, King A. Human sperm deoxyribonucleic acid fragmentation by specific types of papillomavirus. Am J Obstet Gynecol 2001;184:1068-70.

32. Rintala MA, Grenman SE, Pollanen PP, Suominen JJ, Syrjanen SM. Detection of high-risk HPV DNA in semen and its association with the quality of semen. Int J STD AIDS 2004;15:740-3.

33. Motrich RD, Cuffini C, Oberti JP, Maccioni M, Rivero VE. Chlamydia trachomatis occurrence and its impact on sperm quality in chronic prostatitis patients. J Infect 2006;53:175-83.

34. Puerta Suarez J, Sanchez LR, Salazar FC, Saka HA, Molina R, Tissera A, et al. Chlamydia trachomatis neither exerts deleterious effects on spermatozoa nor impairs male fertility. Sci Rep 2017; 7:1126.

35. Gerovassili A, Marcandona O, Asimakopoulos B, Karavasilis V, Panopoulou M, Ikonomidis A. Relationship between ChlamydiaUreaplasma-Mycoplasma genital detection with semen concentration and motility among Greek men. Int J Fertil Steril 2017; 11:130-3.

36. Styler M, Shapiro SS. Mollicutes (mycoplasma) in infertility. Fertil Steril 1985;44:1-12.

37. Bliatka D, Lymperi S, Mastorakos G, Goulis DG. Effect of endocrine disruptors on male reproduction in humans: why the evidence is still lacking? Andrology 2017;5:404-7.

38. Comhaire FH, de Kretser D, Farley TM, Rowe PJ. Towards more objectivity in diagnosis and management of male infertility. Int J Androl 1987;7:1-53.

39. World Health Organization. WHO manual for the standardized investigation and diagnosis of the infertile couple. Geneva: World Health Organization; 2000. 